Nachlas, M.M., Walker, D.G. and Seligmann, A.M.: The histochemical localization of triphosphopyridine nucleotide diaphorase. J. Biophy. Biochem. Cytol., 4: 467-474, 1958.

\title{
Heterogeneity Among Mitochondria in Terms of the Dehydrogenase Activity
}

\author{
Kazuo Ogawa \\ Department of Anatomy, Kansai Medical School, Moriguchi, Osaka
}

In 1963 it was reported in a brief abstract from our laboratory that the TPNH (NADPH) diaphorase activity as demonstrated by the tetrazolium method was positive in the matrix of the pre- and post-synaptic bags located in the superficial layer of the normal adult rat cerebrum. However, not all synapses showed the enzymatic activity and only some of them revealed the positive enzymatic activity indicating the probable existence of the functional heterogeneity among synapses in terms of the diaphorase activity. This heterogeneity was observed among mitochondria in synaptic bags also; a mitochondrion with the positive enzymatic activity was found lying next to another mitochondrion having no activity in a synaptic bag (Anat. Rec., 145, 341, '63).

At that time it was difficult for us to determine whether this heterogeneity observed among organelles is a reflexion of true functional heterogeneity in terms of the enzymatic activity or this heterogenous finding is merely a matter of technical pitfalls. However, thereafter, the similar finding showing heterogeneity in terms of activities of succinic dehydrogenase as well as DPNH (NADH) diaphorase were repeatedly observed among mitochondria in tissues such as the cardiac muscle and kidney in the normal adult rat. The heterogeneity was observed in mitochondria in tissue treated not only as blocks, but also as sections cut by an automatic chopper and further in mitochondrial fractions isolated from homogenates.

It was concluded from these serial findings that there exists the functional heterogeneity among mitochondria in various tissues in terms of the dehydrogenase activity. 